\title{
Single-tablet antiretroviral treatment (once daily)
}

\author{
Nisha Andany MD, Wayne L. Gold MD
}

\section{Single-tablet antiretroviral treatment taken once daily improves adher- ence and quality of life compared with multiple-pill regimens}

Current guidelines recommend combination antiretroviral treatment for all patients with HIV infection. ${ }^{1,2}$ The backbone of this treatment regimen is a dual nucleoside reverse transcriptase inhibitor combination, typically tenofovir/ emtricitabine or abacavir/lamivudine, which is combined with a non-nucleoside reverse transcriptase inhibitor, integrase strand-transfer inhibitor or boosted protease inhibitor. ${ }^{3}$ A meta-analysis of randomized controlled trials (RCTs) showed higher pill burden is inversely associated with adherence to combination antiretroviral treatment and virologic suppression, which provides a rationale for using single-tablet regimens. ${ }^{4}$

Tenofovir/emtricitabine/efavirenz is associated with neuropsychiatric adverse effects

Tenofovir/emtricitabine forms the backbone in three of four single-tablet regimens and is the preferred treatment for HIV/hepatitis B virus coinfection. ${ }^{1}$ Tenofovir/emtricitabine/ efavirenz is recommended only as an alternative regimen, because efavirenz is associated with neuropsychiatric symptoms and may increase suicidality. ${ }^{1,2}$ Tenofovir may cause renal toxicity and osteoporosis. ${ }^{1-3}$

\section{References}

1. Panel on Antiretroviral Guidelines for Adults and Adolescents. Guidelines for the use of antiretroviral agents in HIV-1 infected adults and adolescents. Bethesda (MD): Department of Health and Human Services; 2014. Available: www.aidsinfo.nih.gov/ contentfiles/adultandadolescentgl.pdf (accessed 2015 Oct. 16).

2. Günthard HF, Aberg JA, Eron JJ, et al. Antiretroviral treatment of adult HIV infection: 2014 recommendations of the International Antiviral Society - USA Panel. JAMA 2014;312:410-25.

3. Gandhi M, Gandhi RT. Single-pill combination regimens for treatment of HIV-1 infection. $N$ Engl J Med 2014:371:248-59.

4. Nachega JB, Parienti J, Uthman OA, et al. Lower pill burden and once-daily antiretroviral treatment regimens for HIV infection: a meta-analysis of randomized controlled trials. Clin Infect Dis 2014; 58:1297-307.
Tenofovir/emtricitabine/rilpivirine is less effective in patients with pretreatment HIV viral loads greater than 100000 copies/mL or CD4 counts less than 200 cells/ $\mu \mathrm{L}$

Tenofovir/emtricitabine/rilpivirine should not be prescribed as initial treatment for these patients but is a recommended alternative regimen. ${ }^{1-3}$ Rilpivirine is a non-nucleoside reverse transcriptase inhibitor with less central nervous system toxicity than efavirenz. ${ }^{1}$ Adequate absorption requires administration in conjunction with a high-energy meal (> $400 \mathrm{kcal}) .{ }^{1}$ Rilpivirine should not be prescribed to patients taking proton pump inhibitors and should be prescribed with caution in patients taking other acid-lowering drugs. ${ }^{1,3}$

$C M A J$ invites submissions to "Five things to know about ..." Submit manuscripts online at http://mc.manuscriptcentral .com/cmaj

\begin{abstract}
Abacavir/lamivudine/dolutegravir has the highest genetic barrier to resistance; however, there is a risk of hypersensitivity
\end{abstract}

Abacavir/lamivudine/dolutegravir is recommended as first-line treatment based on RCT evidence. ${ }^{1,2}$ Abacavir is associated with severe hypersensitivity reactions in patients with the HLA-B5701 allele. ${ }^{1,3}$ Pretreatment genetic testing is indicated, and abacavir should be avoided if test results are positive for the presence of the allele., ${ }^{2,3}$ Dolutegravir is an integrase strand-transfer inhibitor with minimal adverse effects and a high genetic barrier to resistance. ${ }^{1-3}$

Tenofovir/emtricitabine/elvitegravir/ cobicistat is well-tolerated but associated with multiple drug interactions

Tenofovir/emtricitabine/elvitegravir/ cobicistat is recommended first-line therapy based on RCT evidence. ${ }^{1}$ It is contraindicated if creatinine clearance is less than $70 \mathrm{~mL} / \mathrm{min}^{1,3}$ Elvitegravir, which is an integrase strand-transfer inhibitor, has a lower genetic barrier to resistance than dolutegravir. ${ }^{1,2}$ Cobicistat is a cytochrome P450 3A4 inhibitor that acts to increase elvitegravir levels in plasma. ${ }^{2}$ However, it has the potential for multiple drug interactions (e.g., with statins and rifampin). ${ }^{1-3}$

\section{Competing interests: None declared.}

This article has been peer reviewed.

Affiliations: Department of Medicine (Andany, Gold), University of Toronto; Division of Infectious Diseases (Gold), University Health Network, Toronto, Ont.

Correspondence to: Wayne Gold, wayne.gold@uhn.ca

CMAJ 2016. DOI:10.1503/cmaj.151412 